\title{
Acerca de la corrosión interna de la envolvente de acero de los hornos rotatorios para fabricar cemento

\section{INTRODUCCION}

En números sucesivos de la revista CEMENTO-HORMIGON han aparecido recientemente estudios y artículos acerca de la corrosión de la envolvente metálica de los hornos rotatorios para fabricar cemento. Así, en el número 416, correspondiente a noviembre de 1968, se publicaba un primer trabajo del Dr. P. BATTI y del Prof. B. TAVASCI (1); un segundo trabajo de los mismos autores apareció en el número 431, de febrero de 1970 (2). Entre ambos trabajos se han publicado otros dos artículos: uno del Dr. Ing. P. PALOMAR COLLADO en el número 418 -enero de 1969- (3) y otro del Dr. D. J. E. VERONELLI en el número 424 -julio de 1969- (4). El artículo del Dr. PALOMAR COLLADO reproducía un trabajo suyo muy anterior, sobre el mismo tema, publicado hace unos 35 años en la citada revista CEMENTO-HORMIGON —número 57, febrero de 1934- (5).

La circunstancia de tener conocimiento personal con varios de los autores precitados, y amistad antigua con algunos de ellos, nos ha movido, aparte del propio interés del tema, a seguir con atención el desarrollo del mismo, así como a caer en la tentación de terciar —en este caso "cuartar" - en el mismo terreno "alzado", "binado" y "terciado" por ellos antes. No obstante, no lo haríamos hasta esperar un posible tercer trabajo de los Dres. TAVASCI y BATTI, en el que dieran la interpretación de los resultados ya obtenidos por ellos, si no creyéramos que en la bibliografía de pasados años se pueden encontrar ya elementos de juicio para interpretar y explicar, al menos parcialmente, el mecanismo de la corrosión interior de la plancha envolvente del horno rotatorio. En lo que sigue se pretende, por lo tanto, exponer un aspecto parcial que creemos pueda servir como una ayuda más en la interpretación de los hechos observados y descritos.

\section{EL FENOMENO DE LA CORROSION}

\subsection{En general}

La corrosión del hierro y del acero consiste, en definitiva, en el paso o transformación de los mismos, del estado metálico y sin carga, al estado iónico (catiónico), como óxidos o como sales. Para ello se precisa el concurso de un agente y de un medio agresivo que 
aporten, bien sea el oxígeno, o bien el correspondiente anión, y humedad en todo caso En tales circunstancias la corrosión tiene lugar formándose un producto primario de la misma, el cual se puede transformar después para dar los productos finales de la corrosión, generalmente complejos. En el caso corriente de la oxidación en medio húmedo y neutro se producen mezclas de oxi-hidróxidos más o menos hidratados y con mayor o menor grado de oxidación, las cuales constituyen la "herrumbre".

\subsection{En el caso particular de la envolvente de los hornos}

Los productos de corrosión que en las circunstancias especiales del horno rotatorio se forman en la parte interior de la envolvente metálica de éste han sido ampliamente observados y descritos. La primera observación detallada fue, en el caso que nos ocupa, la de P. PALOMAR COLLADO (5), la cual, a nuestro juicio, se puede resumir así:

a) la corrosión en un horno rotatorio de vía seca es general en las zonas recubiertas con material aislante entre la plancha y el refractario y casi nula (o insignificante) en las zonas de asiento directo del refractario sobre la plancha;

b) la corrosión es más intensa a medida que se avanza a lo largo del horno, hacia la boca de alimentación, en el sentido de la temperatura decreciente;

c) entre los productos de la corrosión se encuentran sulfato férrico y óxido rojo de hierro - hematites roja (oligisto) $\mathrm{Fe}_{2} \mathrm{O}_{3}$-;

d) como interpretación del fenómeno de corrosión se acepta la oxidación del $\mathrm{SO}_{2}$ de los gases de combustión a $\mathrm{SO}_{3}$, catalizada por el propio óxido de hierro y otros óxidos; la formación de $\mathrm{SO}_{4} \mathrm{H}_{2}$ por hidratación del $\mathrm{SO}_{3}$ y el ataque del ácido sulfúrico a la plancha; la descomposición del sulfato férrico formado, con liberación de $\mathrm{SO}_{2}$ que queda disponible, junto con nuevo $\mathrm{SO}_{2}$ aportado por los gases, para repetir el ciclo.

Por lo que respecta a las dos primeras partes del trabajo de los Dres. BATTI y TAVASCI (1) (2) publicadas hasta el momento, sus minuciosas observaciones y conclusiones se pueden resumir de forma gráfica y esquemática, de acuerdo con la interpretación que de todo ello damos en la figura 1. Estas observaciones complementan y corroboran las hechas por el Dr. PALOMAR, en el sentido de que, cuando en la zona de clinkerización, es decir, en la de máxima temperatura del horno, hay material sílico-aluminoso aislante, interpuesto entre el refractario y la plancha, también se produce corrosión fuerte en ésta. Por lo demás, no hay que perder de vista el hecho de que, a nuestro parecer, la atención de los Dres. TAVASCI y BATTI no se centra sólo en la corrosión (metálica) de la parte interior de la envolvente del horno, sino también, y preferentemente, en todo lo que supone recubrimiento aislante y refractario, y revestimiento interior del horno. En cuanto a los productos de corrosión de la plancha en inmediato contacto con ésta, los autores detectan óxidos ferroso y férrico y principalmente hematites, así como sulfato férrico, de acuerdo con lo observado por el Dr. PALOMAR. Encuentran también sulfatos y cromatos alcalinos en disolución sólida, así como cloruros.

En cuanto a las atinadas observaciones hechas por el Dr. VERONELLI en sus comentarios (4), de ellas se deduce que el agente corrosivo principal de la plancha, tanto en las zonas más calientes como en las menos calientes del horno, es el $\mathrm{SO}_{4} \mathrm{H}_{2}$ formado por hidratación del $\mathrm{SO}_{3}$ a menos de $415^{\circ} \mathrm{C}$, la cual es completa a $200^{\circ} \mathrm{C}$. Los vapores de ácido sulfúrico se condensan al alcanzar el punto de rocío a unos $115^{\circ} \mathrm{C}$. Por otra parte, el $\mathrm{SO}_{3}$ se produce por oxidación del $\mathrm{SO}_{2}$ formado a partir del azufre de los combustibles y a 
favor de la atmósfera oxidante del horno (exceso de $\mathrm{O}_{2}$ ), reacción que se completa a unos $300^{\circ} \mathrm{C}$, o a mayor temperatura en presencia de catalizadores (hasta $550^{\circ} \mathrm{C}$ ), tal como señalaba también el Dr. PALOMAR. Dos puntos muy significativos señala el Dr. VERONELLI: uno (de pasada) es la influencia de los álcalis (sulfatos) volatilizados, punto en el que insistiremos después con mayor detalle; el otro es la influencia del revestimiento interior del horno en el condicionamiento de la temperatura de la chapa a efectos del punto de rocío del ácido sulfúrico, así como la capacidad de adsorción y catálisis de la parte aislante del revestimiento, ayudada o no por eventuales óxidos catalizadores procedentes de las cenizas del combustible (cuando las hay). Así pues, la difusión de gases ( $\mathrm{SO}_{2}, \mathrm{O}_{2}$ y $\mathrm{H}_{2} \mathrm{O}$-vapor-) a través del revestimiento y de los recubrimientos refractario y aislante, la formación catalítica del $\mathrm{SO}_{4} \mathrm{H}_{2}$ en este último y la condensación del ácido sobre la plancha son las causas - y el mecanismo- del ataque y corrosión de la envolvente metálica de los hornos, dando productos que concuerdan con los detectados antaño por el Dr. PALOMAR.

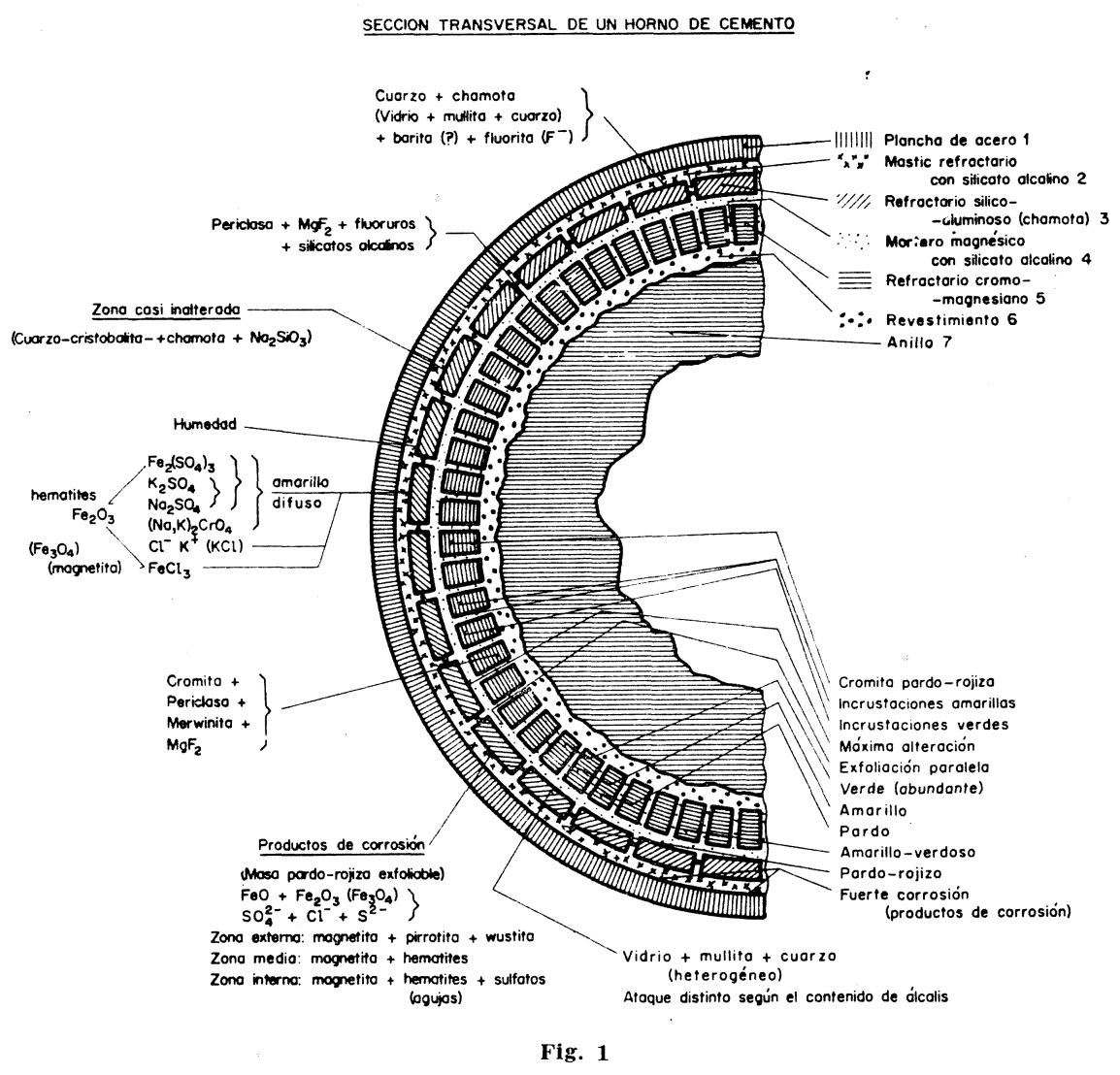

\section{NUESTRA INTERPRETACION}

A primera vista se nos ofrece un medio de compaginar los concordantes resultados y observaciones del Dr. PALOMAR y del Dr. VERONELLI, respectivamente. En efecto, las distintas etapas desde la oxidación del $\mathrm{SO}_{2}$ formado en la combustión, hasta la for- 
mación de hematites roja, como producto final de la corrosión de la envolvente del horno, paso a paso se podrían representar —esquemáticamente- así:

$$
\begin{aligned}
& 6 \mathrm{SO}_{2}+3 \mathrm{O}_{2} \rightleftarrows 6 \mathrm{SO}_{3} \\
& 6 \mathrm{SO}_{3}+6 \mathrm{H}_{2} \mathrm{O} \rightleftarrows 6 \mathrm{SO}_{4} \mathrm{H}_{2} \\
& 4 \mathrm{SO}_{4} \mathrm{H}_{2}+4 \mathrm{Fe} \longrightarrow 4 \mathrm{SO}_{4} \mathrm{Fe}+4 \mathrm{H}_{2} \\
& 4 \mathrm{H}_{2}+2 \mathrm{O}_{2} \rightleftarrows 4 \mathrm{H}_{2} \mathrm{O} \\
& 4 \mathrm{SO}_{4} \mathrm{Fe}+2 \mathrm{SO}_{4} \mathrm{H}_{2}+\mathrm{O}_{2} \longrightarrow 2\left(\mathrm{SO}_{4}\right)_{3} \mathrm{Fe}_{2}+2 \mathrm{H}_{2} \mathrm{O} \\
& 2\left(\mathrm{SO}_{4}\right)_{3} \mathrm{Fe}_{2} \longrightarrow 2 \mathrm{Fe}_{2} \mathrm{O}_{3}+6 \mathrm{SO}_{3} \\
& 6 \mathrm{SO}_{3} \rightleftarrows 6 \mathrm{SO}_{2}+3 \mathrm{O}_{2}
\end{aligned}
$$

En estas siete etapas convencionales se observa que la primera y la última son iguales y de sentido contrario, lo que explica y justifica el ciclo que intuía el Dr. PALOMAR en su primer trabajo. Pero, además, sumados miembro a miembro los siete equilibrios y reacciones de desplazamiento anteriores, se obtiene como resultado:

$$
4 \mathrm{Fe}+3 \mathrm{O}_{2} \longrightarrow 2 \mathrm{Fe}_{2} \mathrm{O}_{3}
$$

que indica a las claras que la hematites roja u óxido férrico es el producto final de la oxidación de la chapa. Estados intermedios del proceso de corrosión pueden incluir también, entre los productos de la misma, el sulfato férrico según [5] o incluso el sulfato ferroso según [3], y tal vez otros compuestos ferrosos, férricos o intermedios (magnetita $\mathrm{Fe}_{3} \mathrm{O}_{4}$ ), todo ello de acuerdo con las observaciones hechas por los Dres. BATTI y TAVASCI. Las reacciones y equilibrios [1] a [7] se pueden considerar como ecuaciones químicas a efectos de sumación miembro a miembro porque, salvo el [1] y el [7] que se eliminan entre sí, el [2] y el [4] están muy desplazados hacia la derecha; el [2] por acción catalítica y condensación del ácido sulfúrico gaseoso que se sustrae así al equilibrio; y el [4] por eliminación del agua, según la propia reacción [2].

Por otra parte, sin catalizadores, el equilibrio [2] a $300^{\circ} \mathrm{C}$ está apreciablemente desplazado hacia la izquierda (disociación), a $350^{\circ} \mathrm{C}$ la disociación es del orden del $50 \%$ y a $420^{\circ} \mathrm{C}$ es casi completa. El equilibrio [4], a su vez, presenta una disociación prácticamente nula a $540^{\circ} \mathrm{C}$, del orden del $0,5 \%$ a $1.100^{\circ} \mathrm{C}$ y del $15 \%$ a $1.650^{\circ} \mathrm{C}$, en condiciones ordinarias.

Se podría considerar también, en cierta medida y determinadas condiciones, la oxidación del hierro a través de la acción del vapor de agua según:

$$
\begin{aligned}
4 \mathrm{Fe}+6 \mathrm{H}_{2} \mathrm{O} & \rightleftarrows 2 \mathrm{Fe}_{2} \mathrm{O}_{3}+6 \mathrm{H}_{2} \\
6 \mathrm{H}_{2}+3 \mathrm{O}_{2} & \rightleftarrows 6 \mathrm{H}_{2} \mathrm{O}
\end{aligned}
$$

equilibrios que se hallarían muy desplazados hacia la derecha, conduciendo al mismo resultado:

$$
4 \mathrm{Fe}+3 \mathrm{O}_{2} \longrightarrow 2 \mathrm{Fe}_{2} \mathrm{O}_{3}
$$

De un modo análogo a la formación de sulfatos ferroso y férrico se podría considerar la formación de cloruros ferroso y férrico, en el caso en que los gases del horno contu- 
viesen cloro, o en el caso en que por una circunstancia cualquiera llegasen cloro o cloruros a ponerse en contacto con la plancha. (A este respecto, el Dr. PALOMAR señala que el aislante interpuesto entre ésta y el refractario, en el caso de corrosión que él describió, estaba húmedo por haberse mojado durante el transporte por barco; si la mojadura fue producida por agua de mar, evidentemente el material aislante quedó impregnado de sales, y en particular de cloruros, lo cual contribuiría en gran medida a facilitar y acelerar la corrosión.)

En cuanto a la existencia de cromatos alcalinos y de cromita (cromato férrico) puesta de relieve por los Dres. TAVASCI y BATTI, se explica fácilmente por la interacción de los álcalis - difundidos como se verá después a través del revestimiento y del recubrimiento refractario- y de los compuestos férricos formados por corrosión - difundidos en sentido contrario-, sobre los ladrillos cromo-magnesianos. Tampoco ofrece dificultad de explicación en este sentido la presencia de compuestos magnésicos, tales como periclasa y merwinita, así como de cuarzo, mullita, etc., ni las coloraciones apreciadas por los mencionados autores.

Nuestra atención se centra en los álcalis, en los sulfatos (alcalinos y férrico) y en el óxido férrico. El mecanismo para explicar la formación y presencia de óxido férrico, de sulfato férrico y de eventuales compuestos ferrosos, según nuestro esquema anterior, responde a una doble interpretación, a saber: en las zonas más calientes del horno, rebasada hacia atrás la zona de clinkerización, es la simple difusión de los gases $\mathrm{SO}_{2}, \mathrm{O}_{2}, \mathrm{SO}_{3}$ y $\mathrm{H}_{2} \mathrm{O}$ (vapor) a través del revestimiento, del forro refractario y del recubrimiento aislante (en su caso), la que aporta el elemento corrosivo $\mathrm{SO}_{4} \mathrm{H}_{2}$ al contacto con la plancha; en las partes menos calientes del horno, más hacia atrás todavía, y donde se producen anillos de sulfatos, es la penetración y difusión de los sulfatos alcalinos a través del revestimiento, del refractario y del aislante lo que lleva los agentes corrosivos a la chapa.

\subsection{Corrosión en las zonas calientes}

En la zona de clinkerización, entre $1.400^{\circ} \mathrm{C}$ y $1.500^{\circ} \mathrm{C}$, según el equilibrio expuesto por el Dr. VERONELLI

$$
2 \mathrm{SO}_{2}+\mathrm{O}_{2} \stackrel{\mathrm{T} \cdot 21.200^{\circ} \mathrm{C}}{\stackrel{\mathrm{T}<1.200^{\circ} \mathrm{C}}{\rightleftarrows}} 2 \mathrm{SO}_{3} ; \quad \frac{\left[\mathrm{SO}_{3}\right]}{\left[\mathrm{SO}_{2}\right]}=\mathrm{K} \sqrt{\left[\mathrm{O}_{2}\right]}
$$

apenas existiría $\mathrm{SO}_{3}$ formado, y máxime si la combustión no se verifica en condiciones francamente oxidantes (exceso de aire $-\mathrm{y}$ de $\mathrm{O}_{2}-$ ). Por otra parte, el rendimiento en $\mathrm{SO}_{3}$ de la reacción [12] en condiciones ordinarias es muy escaso a más de $950^{\circ} \mathrm{C}$ y a menos de $200^{\circ} \mathrm{C}$, siendo menor cuanto más alta es la temperatura y mayor cuanto más baja, dentro de dicho intervalo, existiendo un óptimo alrededor de $400^{\circ}$ a $450^{\circ} \mathrm{C}$, pero siendo, de todos modos, la reacción muy lenta. La constante $\mathrm{K}_{\mathrm{p}}=\mathrm{p}_{\mathrm{SO}_{3}}^{2} / \mathrm{p}_{\mathrm{SO}_{2}}^{2} \cdot \mathrm{p}_{\mathrm{O}_{2}}$ (dada en presiones parciales), expresada en fracciones molares sería $\mathrm{Kp}=\mathrm{N} \cdot \mathrm{n}^{2}{ }_{\mathrm{SO}_{3}} / \mathrm{n}^{2}{ }_{\mathrm{SO}_{2}} \cdot \mathrm{n}_{\mathrm{O}_{2}} \cdot \mathrm{P}$, donde $\mathrm{P}$ es la presión total y $\mathrm{N}$ el número total de moles. De ella se deduce que:

$$
\frac{\mathrm{n}_{\mathrm{SO}_{3}}}{\mathrm{n}_{\mathrm{SO}_{2}}}=\sqrt{\mathrm{K}_{\mathrm{p}} \cdot \mathrm{P} \cdot \frac{\mathrm{n}_{\mathrm{O}_{2}}}{\mathrm{~N}}}
$$


con lo que la presencia de gases inertes o ajenos al equilibrio [12], al hacer aumentar $\mathrm{N}$ rebajarían aún más el rendimiento en $\mathrm{SO}_{3}$. En las zonas de combustión y clinkerización es donde mayor concentración de gases hay, y por lo tanto donde mayor es $\mathrm{N}$, un motivo más para la escasísima formación y presencia de $\mathrm{SO}_{3}$ en dichas zonas.

De lo anterior se deduce que debe existir a lo largo del horno, y más arriba de la zona le clinkerización, otra zona a una temperatura óptima para la formación y existencia de $\mathrm{SO}_{3}$. En esta parte del horno puede tener también lugar la formación de $\mathrm{SO}_{3}$ por descomposición de los sulfatos de metales poco fusibles y fuertemente básicos (alcalinos y de calcio), si acompañan al crudo, uniéndose (o no) la base a otros anhídridos menos volátiles que el $\mathrm{SO}_{3}$ :

$$
\begin{aligned}
\mathrm{SO}_{4} \mathrm{Ca}+\mathrm{SiO}_{2} & \longrightarrow \mathrm{SiO}_{3} \mathrm{Ca}+\mathrm{SO}_{3} \\
2 \mathrm{SO}_{4} \mathrm{Ca}+2 \mathrm{SiO}_{2} & \longrightarrow 2 \mathrm{CaO} \cdot \mathrm{SiO}_{2}+2 \mathrm{SO}_{2}+\mathrm{O}_{2} \\
\mathrm{SO}_{4} \mathrm{Ca} & \rightleftarrows \mathrm{CaO}+\mathrm{SO}_{3}\left(\mathrm{SO}_{2}+\mathrm{O}\right) \\
\left(\mathrm{SO}_{4}\right)_{3} \mathrm{Fe}_{2} & \longrightarrow \mathrm{Fe}_{2} \mathrm{O}_{3}+3 \mathrm{SO}_{3}
\end{aligned}
$$

La reacción de descomposición [15], por ejemplo, es casi completa a $1.375^{\circ} \mathrm{C}$ mientras que a $800^{\circ}-1.000^{\circ} \mathrm{C}$ la descomposición del $\mathrm{SO}_{4} \mathrm{Ca}$ es insignificante. También puede verificarse la descomposición de eventuales pirosulfatos, con igual resultado:

$$
\mathrm{S}_{2} \mathrm{O}_{7} \mathrm{~K}_{2} \rightleftarrows \mathrm{SO}_{4} \mathrm{~K}_{2}+\mathrm{SO}_{3}
$$

Por lo tanto, en la mencionada zona y a partir de ella puede tener lugar la difusión del $\mathrm{SO}_{3}$, junto con la de los otros gases y vapores antes mencionados, a través de las distintas capas internas del horno, para dar lugar a la corrosión de la chapa, según el esquema de las reacciones [1] a [7].

\subsection{Corrosión en las zonas menos calientes}

$\mathrm{El} \mathrm{SO}_{2}$ y el $\mathrm{SO}_{3}$ formados y no difundidos a través de las distintas capas de recubrimiento, ni expulsados con los gases por la chimenea, son captados por el crudo al extremo del horno, o condensados en el polvo del horno y de la chimenea, y forman sulfato cálcico y sulfatos alcalinos. Un posible mecanismo para explicar la formación de sulfato cálcico en las partes menos calientes del horno, semejante al del grupo de reacciones [1] a [7] podría ser el constituido por las reacciones [18] a [21].

$$
\begin{aligned}
8 \mathrm{CaO}+8 \mathrm{SO}_{2} & \longrightarrow 8 \mathrm{SO}_{3} \mathrm{Ca} \\
8 \mathrm{SO}_{3} \mathrm{Ca} & \longrightarrow 6 \mathrm{SO}_{4} \mathrm{Ca}+2 \mathrm{SCa} \\
2 \mathrm{SCa}+4 \mathrm{SO}_{2} & \longrightarrow 2 \mathrm{SO}_{4} \mathrm{Ca}+4 \mathrm{~S} \\
4 \mathrm{CaO}+4 \mathrm{~S} & \longrightarrow \mathrm{SO}_{4} \mathrm{Ca}+3 \mathrm{SCa}
\end{aligned}
$$

Este grupo de reacciones sumadas, así como la [18] y la [19] por una parte, o la [20] y la [21], por otra, dan por resultado el equilibrio preconizado por VOGEL (6):

$$
4 \mathrm{CaO}+4 \mathrm{SO}_{2} \longmapsto 3 \mathrm{SO}_{4} \mathrm{Ca}+\mathrm{SCa}
$$


La reacción [18] se verifica a temperaturas superiores a $300^{\circ} \mathrm{C}$, pero a temperaturas aún mayores tiene ya lugar la [19]. En cualquier caso, el equilibrio heterogéneo [22] se halla desplazado hacia la izquierda a temperaturas superiores a $1.045^{\circ} \mathrm{C}$ (proceso de fabricación de cemento y de ácido sulfúrico a partir de yeso), y hacia la derecha a temperaturas inferiores a $1.045^{\circ} \mathrm{C}$, que es lo que se supone pasa en el horno de cemento. De este modo, entre $700^{\circ}$ y $1.000^{\circ} \mathrm{C}$, o estrechando más, entre $900^{\circ}$ y $1.000^{\circ} \mathrm{C}$, tiene lugar la máxima formación de sulfato cálcico, dependiendo mucho de la concentración de $\mathrm{SO}_{2}$ en los gases, la cual en determinada zona del horno, es mayor que la calculada a partir del azufre del combustible y de los sulfatos del crudo, aumentando más y más hasta que se alcanza un equilibrio, generalmente a partir de los 35 metros de la boca de salida del horno. La reacción [21] es completa - y por lo tanto todo el complejo [18] a [21], es decir, el equilibrio [22], tiene mayores probabilidades de realidad-a condición de que no haya oxidación del azufre, esto es, de que reinen condiciones reductoras en el horno. Como esto, en general, no es así, quiere decirse que la relación $\mathrm{SO}_{4} \mathrm{Ca} / \mathrm{SCa}$ puede tomar distintos valores, dependiendo de la temperatura y de las condiciones (oxidantes o reductoras) del medio. El equilibrio [22] contribuye a la formación de sulfatos, y particularmente de sulfato cálcico, pero no es el solo determinante de dicha formación. Por otra parte, el polvo en la atmósfera del horno favorece su desplazamiento hacia la derecha, así como la temperatura decreciente. Sin embargo, la reacción [22] se verifica por difusión, la cual es más activa al aumentar la temperatura; de aquí que también en cuanto al factor temperatura exista un compromiso entre el desplazamiento del equilibrio hacia la izquierda y la activación de la difusión.

Los sulfatos alcalinos y cálcicos bajan de nuevo con el material crudo a lo largo del horno, hasta descomponerse o reaccionar según [13] a [17], en las zonas de temperatura alta, volviendo atrás los productos de la descomposición para ser captados de nuevo por condensación en las partes más frías, y cerrándose así el ciclo. De este modo, el contenido de azufre (sulfatos) del crudo experimenta un enriquecimiento local, siendo máximo en una zona determinada del horno y, correspondientemente, mínimo $\left(e l \mathrm{SO}_{3}\right)$ en la fase gaseosa. En esta zona, por lo tanto, no sería muy operante el mecanismo aceptado según 3 y 3.1 para explicar la corrosión de la envolvente.

Parece, en cambio, más aceptable como explicación, el hecho de la segregación de compuestos por vaporización y condensación, según las ideas que sirvieron a VOGEL para explicar la formación de anillos de crudo en los hornos rotatorios. Los más genuinos representantes de estos compuestos son los de los álcalis que, procedentes de las materias primas - componentes arcillosos-, con el $\mathrm{SO}_{2}$ del azufre del combustible dan lugar a sulfatos, según:

$$
\mathrm{K}_{2} \mathrm{O}+\mathrm{SO}_{2}+\frac{1}{2} \mathrm{O}_{2} \rightleftarrows \mathrm{SO}_{4} \mathrm{~K}_{2}
$$

equilibrio cuya constante viene expresada por:

$$
\mathrm{K}_{1}=\left[\mathrm{SO}_{4} \mathrm{~K}_{2}\right] /\left[\mathrm{K}_{2} \mathrm{O}\right] \cdot\left[\mathrm{SO}_{2}\right] \cdot\left[\mathrm{O}_{2}\right]^{1 / 2}
$$

Dada la escasa presión de vapor del sulfato potásico, éste constituye una fase sólida en las partes menos calientes del horno, con lo cual su concentración en la fase gaseosa se puede considerar muy pequeña y constante. Así, pues, $\mathrm{K}_{2}=1 /\left[\mathrm{K}_{2} \mathrm{O}\right] \cdot\left[\mathrm{SO}_{2}\right] \cdot\left[\mathrm{O}_{2}\right] 1 / 2$, 
de tal manera que la concentración de sulfatos alcalinos en la fase gaseosa depende de la concentración de $\mathrm{SO}_{2}$ en la misma, siendo tanto menor aquélla cuanto mayor es ésta. El equilibrio [23] queda ligado al equilibrio [12] a través del $\mathrm{SO}_{2}$ en la fase gaseosa, de modo que $\mathrm{K}_{3}=1 /\left[\mathrm{K}_{2} \mathrm{O}\right]\left[\mathrm{SO}_{3}\right]$. Por lo tanto, la condensación de los álcalis como sulfatos en forma sólida crece con la concentración de azufre oxidado en la fase gaseosa. Lo mismo sucede con el $\mathrm{SO}_{4} \mathrm{Ca}$, como pone de relieve de forma directa el equilibrio [15].

El punto de fusión del sulfato potásico es $1.076^{\circ} \mathrm{C}$ y el del eutéctico $\mathrm{SO}_{4} \mathrm{~K}_{2} \cdot \mathrm{SO}_{4} \mathrm{Ca}, 867^{\circ}$ centígrados. Quiere esto decir que por encima de $867^{\circ} \mathrm{C}$ (y más aún por encima de $1.076^{\circ} \mathrm{C}$ ) puede haber sulfatos fundidos, como aerosoles en la fase gaseosa del horno, los cuales se condensan en zonas de temperatura inferior a la mínima de las citadas.

Esquematizando con una visión personal las ideas de VOGEL acerca de la formación de anillos de crudo, la figura 2 permite apreciar el mecanismo del paso de álcalis (sulfatos) de la fase gaseosa a las paredes del horno, a través de los gases o del polvo. En el punto 1 del horno los sulfatos alcalinos se vaporizan, por ser su presión del vapor $\mathrm{p}_{1}$ suficientemente alta a la temperatura correspondiente $\mathrm{T}_{1}$. En forma de vapor pasan así a la fase gaseosa, en la que a la temperatura $\mathrm{T}_{2}<\mathrm{T}_{1}$ ejercen una presión $\mathrm{p}_{2}<\mathrm{p}_{1}$, siendo arrastrados por el flujo gaseoso en la dirección de éste y de los gradientes descendientes de temperatura. Más adelante, a una temperatura $\mathrm{T}_{3}<\mathrm{T}_{2}$, del orden de la mayor de las indicadas antes, los sulfatos alcalinos se condensan en forma de aerosol con una presión de vapor $\mathrm{p}_{3}<\mathrm{p}_{2}$.

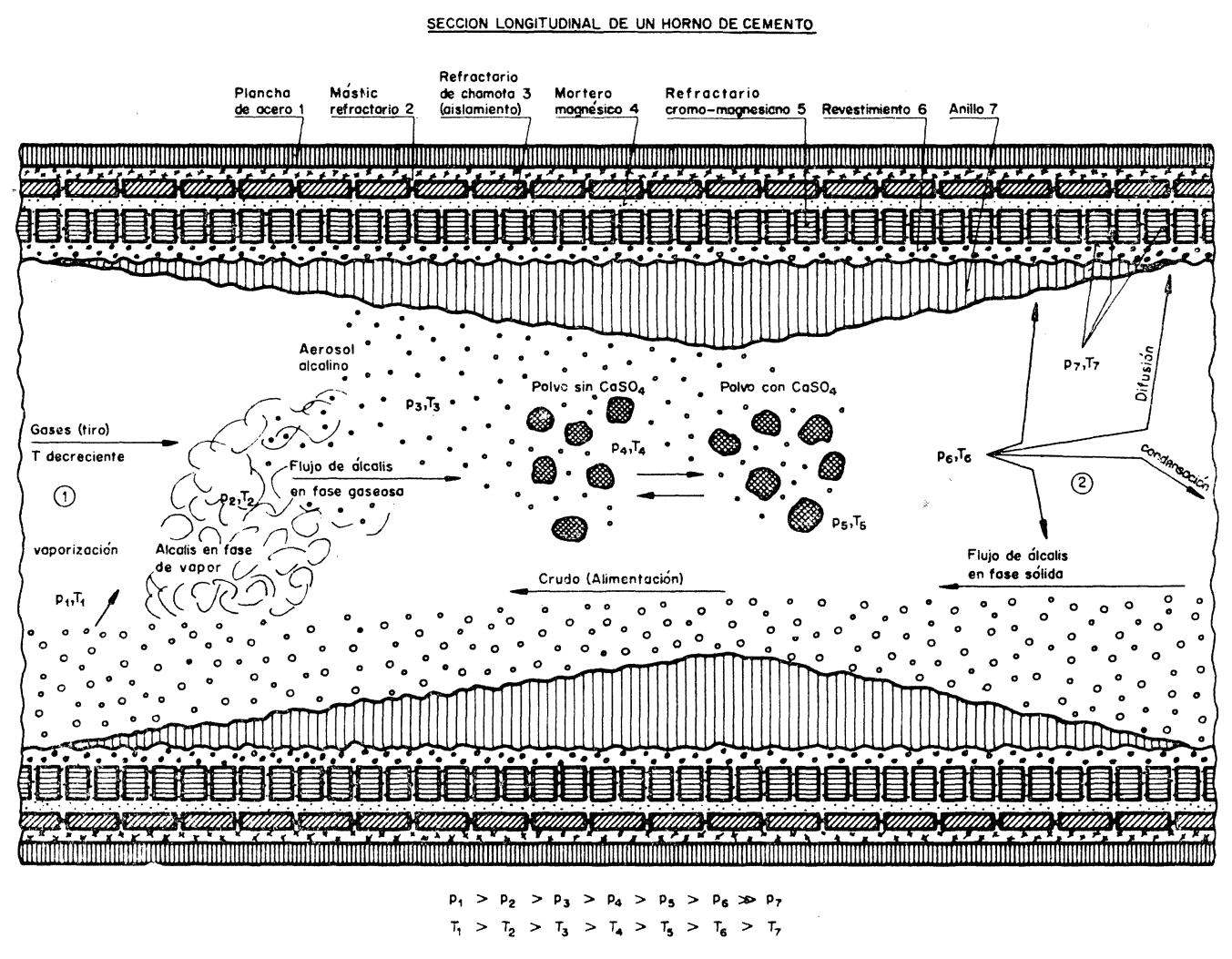

Fig. 2 
En esta misma zona y más adelante, los sulfatos alcalinos, ya en forma de aerosol, se ponen en contacto con partículas finísimas de polvo flotante en la atmósfera del horno, sobre las que se condensan, ya que estas partículas se hallan a una temperatura $\mathrm{T}_{4}<\mathrm{T}_{3}$ y la presión de vapor de los sulfatos condensados sobre ellas es $\mathrm{p}_{4}<\mathrm{p}_{3}$. La diferencia $\mathrm{p}_{3}-\mathrm{p}_{4}$ es pequeña, pero capaz a pesar de ello de producir una destilación, incluso isotérmica, dada la diferencia de grado de división de los sulfatos alcalinos en el aerosol y en el polvo. Así crecen las gotas grandes a costa de las pequeñas, pues éstas tienen mayor presión de vapor, y aumenta la condensación del aerosol.

El polvo considerado se supone exento de sulfato cálcico. Pero el mismo fenómeno de condensación tiene lugar sobre partículas que lo contienen, lo cual puede suceder más adelante en el horno, a una temperatura $\mathrm{T}_{5}<\mathrm{T}_{4}$, del orden de la segunda de las señaladas anteriormente. En estas partículas la presión de vapor de los sulfatos alcalinos será $\mathrm{p}_{5}<\mathrm{p}_{4}$, pero la diferencia $\mathrm{p}_{4}-\mathrm{p}_{5}$ es en este caso mayor, ya que la disolución de $\mathrm{SO}_{4} \mathrm{Ca}$ en $\mathrm{SO}_{4} \mathrm{~K}_{2}$ para formar el eutéctico rebaja el punto de fusión del $\mathrm{SO}_{4} \mathrm{~K}_{2}$, y por lo tanto también su presión de vapor.

A partir de este punto del horno y hacia arriba - punto 2- se depositan los sufatos alcalinos (y de calcio) sobre el crudo $\left(\mathrm{p}_{6}, \mathrm{~T}_{6}\right)$ y también sobre el revestimiento y el refractario. En efecto, en este último, a la temperatura $\mathrm{T}_{7}<\mathrm{T}_{6}$, los álcalis se encuentran como silicatos y aluminatos y su presión de vapor $\mathrm{p}_{7} \ll \mathrm{p}_{6}$ es insignificante frente a la de los sulfatos. Así, pues, la diferencia $\mathrm{p}_{6}-\mathrm{p}_{7}$ es muy grande y ello provoca la precipitación definitiva. Por contacto y difusión, ya entre sólidos, los sulfatos alcalinos se fijan y penetran a través del revestimiento, del forro refractario y del aislamiento. Las consiguientes pegaduras, si crecen, pueden dar lugar a un anillo de contención de crudo, pero éste es un problema que en este caso no nos interesa como le interesó a VOGEL en el suyo.

Siguiendo a este autor, ya dentro del conjunto revestimiento-refractario-aislante, la concentración de sulfatos alcalinos tiene un máximo muy pronunciado en un cierto lugar, en función de la distancia; máximo que se explica del siguiente modo: consideremos ahora otro corte transversal del horno (figura 3); en la atmósfera de éste y en la sección considerada, existe un gradiente continuo de temperatura desde $\mathrm{T}_{0}$ (atmósfera del horno) hasta $\mathrm{T}_{4}$ (masa refractaria). En esa dirección se difunden desde el horno vapores y fundidos, y en particular sulfatos alcalinos. La concentración de éstos en el anillo y en el revestimiento (de $\mathrm{K}_{1}$ a $\mathrm{K}_{2}$ ) es mayor que en los gases del horno, pues al existir un gradiente decreciente de temperatura de $\mathrm{T}_{0}$ a $\mathrm{T}_{1}$ (antes de $\mathrm{T}_{6}$ a $\mathrm{T}_{7}$-fig. 2-) se produce condensación, al ser también menor la presión de vapor de los sulfatos alcalinos en el revestimiento que en la atmósfera del horno $\left(\mathrm{p}_{7} \ll \mathrm{p}_{6}\right.$-fig. 2-), por la disolución de $\mathrm{SO}_{4} \mathrm{Ca}$ en $\mathrm{SO}_{4} \mathrm{~K}_{2}$. Del fundido $\mathrm{SO}_{4} \mathrm{Ca} \cdot \mathrm{SO}_{4} \mathrm{~K}_{2}$ se evapora el $\mathrm{SO}_{4} \mathrm{~K}_{2}$ y se condensa en una zona del refractario correspondiente a una determinada caída de temperatura a $\mathrm{T}_{3}$, a la que corresponde la máxima concentración de álcalis (sulfatos alcalinos). El sulfato potásico por interacción con la chamota del refractario se descompone, cediendo $\mathrm{SO}_{2}$ y formando aluminatos y silicatos alcalinos que se difunden más hacia adentro del ladrillo, pero más despacio, por lo que la curva de distribución de los álcalis con la distancia tiene forma de campana. Al máximo de álcalis (expresados como óxidos) no corresponde el máximo de sulfatos, en virtud de la citada descomposición, y el $\mathrm{SO}_{2}$ formado en ella, por su mayor difusibilidad y estando muy próximo ya a la plancha de la envolvente, puede llegar al contacto con ésta.

Por difusión gaseosa análoga pueden llegar a la chapa oxígeno y vapor de agua, y en- 
tonces estamos de nuevo en el caso del proceso cíclico [1] a [7] que da lugar al ataque y corrosión de la misma. Naturalmente que esta corrosión será más fácil e intensa en aquellas zonas separadas de la clinkerización. en las que por razón de las temperaturas tiene lugar el ciclo de vaporización y condensación de los sulfatos alcalinos y de calcio. En estas zonas el revestimiento es también más permeable, lo cual facilita la más rápida difusión de los fundidos y de los gases y vapores hacia la chapa de la envolvente. La formación de ácido sulfúrico y su condensación por debajo de su punto de rocío sobre la chapa hace lo demás, desde el punto de vista de la corrosión de ésta.

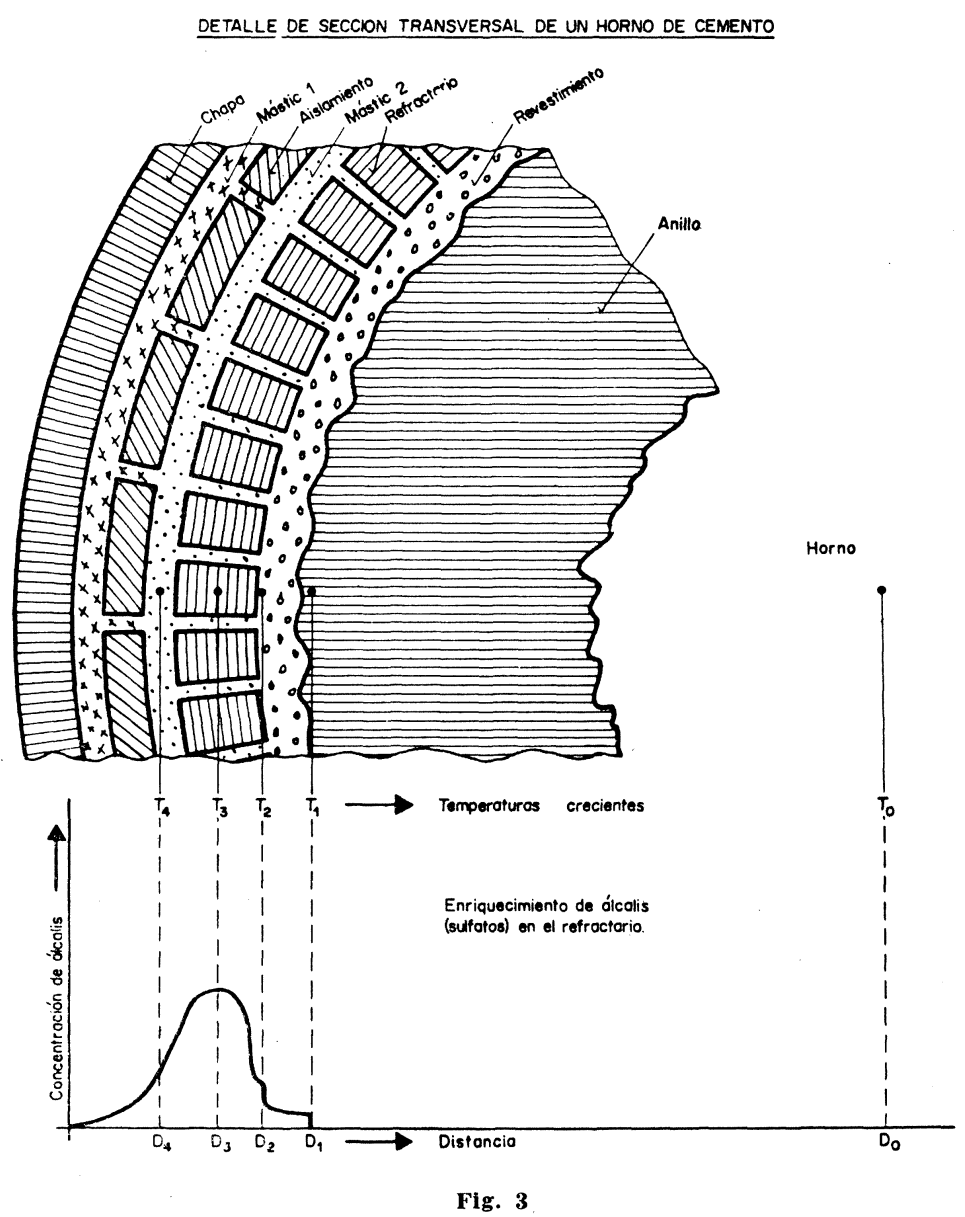

En resumen: la fijación de $\mathrm{SO}_{2}\left(\mathrm{SO}_{3}\right)$ por los álcalis del crudo (materias primas -arcillas-) con formación de sulfatos alcalinos y de calcio que se vaporizan y condensan de forma cíclica en una determinada zona del horno; la difusión de estos sulfatos a través del revestimiento y del recubrimiento del horno, y su concentración en una determinada zona del refractario; su descomposición con formación de $\mathrm{SO}_{2}$, el cual sigue difundiéndose hacia la plancha envolvente; la formación de ácido sulfúrico por posible catálisis de adsorción, y su condensación sobre la plancha, creemos que explican suficientemente su corrosión, en virtud de los mecanismos cuyos posibles detalles hemos descrito. 


\section{CONSIDERACIONES FINALES}

De suceder las cosas así, o de modo análogo, es fácil sacar una serie de consecuencias acordes con las ideas expuestas.

Todas ellas se pueden referir directamente a las condiciones favorables para la corrosión interna de la envolvente del horno, e indirectamente a los remedios y acciones para evi. tarla.

Se podrían enumerar las siguientes, sin carácter exhaustivo:

a) la corrosión parece tener lugar preferentemente (pero no exclusivamente) en las zonas del horno más apartadas de la zona de clinkerización;

b) en todo caso, parece ser que la existencia de un aislamiento entre la envolvente del horno y el recubrimiento refractario favorece la corrosión, tal vez por favorecer la condensación del ácido sulfúrico formado en virtud de los mecanismos anteriormente descritos;

c) favorece la corrosión la posibilidad de difusión de los gases, vapores y fundidos a través del revestimiento, del recubrimiento refractario y del aislante, si lo hay (en tal sentido, la mayor inercia química del refractario de magnesita y cromo-magnesita, por ejemplo, asi como sus mayores variaciones dimensionales, pueden favorecer la corrosión, más que la mayor reactividad de otros -aluminosos y sílico-aluminosos, por ejemplo- ya que los primeros permiten la más fácil difusión);

d) la presencia de álcalis en las materias primas y la de azufre en el combustible contribuyen, cada una en su medida, a favorecer la corrosión (empleo de lignitos o fuel-oil con contenidos altos de azufre);

e) por la misma razón, la corrosión puede ser más fácil, intensa y extensa en hornos largos que en cortos, y en los de vía húmeda que en los de vía seca, al ser mayor en aquéllos la cantidad de álcalis y sulfatos recuperados;

f) también por la misma razón la corrosión parece ser más fácil en los hornos Lepol, dentro de los de vía seca;

g) en cualquier caso, la corrosión será mayor cuanto más álcalis y sulfatos alcalinos y de calcio se recuperen y reincorporen al crudo de alimentación;

h) también la corrosión puede ser mayor cuanto mayor sea la cantidad de polvo que se forme en el horno (aptitud del crudo para la granulación y estabilidad de los gránulos);

i) la atmósfera oxidante del horno favorece la corrosión de la envolvente, si bien una atmósfera reductora puede tener otros inconvenientes, y entre ellos la mayor facilidad para la formación de anillos (se debe trabajar con el mínimo exceso posible de aire y ejercer un control riguroso de la combustión);

j) favorece también la corrosión interior de la envolvente la facilidad de entrada de humedad desde el exterior, lo cual es más probable en los hornos roblonados que en los enteramente soldados, pues los primeros nunca son del todo impermeables; la penetración de agua contribuye también a destruir el recubrimiento refractario;

k) el desconchado de los ladrillos refractarios favorece, en principio, la facilidad de acceso de los gases y vapores corrosivos a la envolvente. El desconchado se produce 
por infiltración de fases líquidas y por recristalizaciones del propio ladrillo, al ser sometido a temperaturas superiores a la suya propia de cocción; se evita esto, no precisamente interponiendo aislantes térmicos entre la plancha envolvente y el refractario - con lo que éste se calentaría más-, sino por el contrario, facilitando el paso del calor a través del refractario, con detrimento del rendimiento térmico (en tal sentido serían recomendables recubrimientos refractarios de espesor mínimo); pero así se favorece también el acceso de agentes corrosivos hacia la envolvente, por lo cual existe un compromiso entre rendimiento térmico, duración y estabilidad del refractario y corrosión de la envolvente, que sólo se puede resolver satisfactoriamente a base de un revestimiento firme, adherente, compacto y de grosor adecuado, del propio material crudo que se cuece.

Creemos que cuanto antecede, que corrobora y amplía lo observado y expuesto sobre el tema por los autores mencionados en las referencias bibliográficas, puede constituir una aportación más, pero no la última, para la interpretación de los hechos y la explicación de los mecanismos concernientes a la corrosión interna de la envolvente de acero de los hornos rotatorios para cemento.

\section{REFERENCIAS}

(1) Batri, P., y Tavasci, B.: "Estudio sobre la corrosión del revestimiento de un horno rotatorio". Cemento-Hormigón. Núm. 416, 1015-1026, noviembre 1968.

(2) Batti, P., y TavascI, B.: "Estudio sobre la corrosión del revestimiento de un horno rotatorio". Nota II. Cemento-Hormigón. Núm. 431, 127-137, febrero 1970.

(3) Palomar Collado, P.: "Un fenómeno de corrosión en horno rotatorio de cemento". Cemento-Hormigón. Núm. 418, 43-49, enero 1969.

(4) Veronelli, D. J. E.: "La corrosión de la envolvente metálica de hornos rotatorios de cemento portland: Un aporte al esclarecimiento del problema”. Cemento-Hormigón. Núm. 424, 759-762, julio 1969.

(5) Palomar Collado, P.: "Un fenómeno de corrosión en horno rotatorio de cemento". Cemento-Hormigón. Núm. 57, febrero 1934.

(6) VogeL, E.: “Zum Problem der Stauringbildung in Zementdrehofen”. Silikattechnik. 9, 361, 449, 502 (1958). 